\title{
AU DELÀ DU «HUB » GÉOPOLITIQUE, LA RÉGULATION CONTESTÉE DU SECTEUR ÉNERGÉTIQUE EN TURQUIE
}

L'exemple du marché du gaz naturel

Elvan Arik et Elshan Mustafayev

\section{L'Harmattan | Confluences Méditerranée}

\section{$2014 / 4-N^{\circ} 91$ \\ pages 53 à 66}

ISSN 1148-2664

Article disponible en ligne à l'adresse:

http://www.cairn.info/revue-confluences-mediterranee-2014-4-page-53.htm

Pour citer cet article

Arik Elvan et Mustafayev Elshan, « Au delà du « hub » géopolitique, la régulation contestée du secteur énergétique en Turquie » L'exemple du marché du gaz naturel,

Confluences Méditerranée, 2014/4 º 91, p. 53-66.

Distribution électronique Cairn.info pour L'Harmattan.

(c) L'Harmattan. Tous droits réservés pour tous pays.

La reproduction ou représentation de cet article, notamment par photocopie, n'est autorisée que dans les limites des conditions générales d'utilisation du site ou, le cas échéant, des conditions générales de la licence souscrite par votre établissement. Toute autre reproduction ou représentation, en tout ou partie, sous quelque forme et de quelque manière que ce soit, est interdite sauf accord préalable et écrit de l'éditeur, en dehors des cas prévus par la législation en vigueur en France. II est précisé que son stockage dans une base de données est également interdit. 


\section{Au delà du " hub » géopolitique, la régulation contestée du secteur énergétique en Turquie L'exemple du marché du gaz naturel}

Dans l'optique de devenir un hub gazier, depuis le début des années 2000, la Turquie a engagé d'importantes réformes structurelles de ses marchés énergétiques internes. Traditionnellement, seules des analyses géopolitiques servent à décrire le hub gazier turc. Après un rappel des principaux enjeux énergétiques régionaux, cet article propose une analyse de la situation interne à la Turquie puis met en perspective les contradictions et les potentiels impacts d'une régulation économique et politique ambivalente du secteur gaz naturel de la part du parti au pouvoir. Ce décentrement scalaire et thématique permet au final de compléter et de réévaluer la pertinence de cette notion de hub appliquée au cas turc.

$\sim$

e serait au début des années 1990 pour certains, au moment de la chute de l'URSS et du repositionnement de la Turquie dans une nouvelle aire d'influence, ou pour d'autres, deux décennies plus tard, à la fin des années 2000, avec la concrétisation de la nouvelle politique étrangère du ministre Davutoğlu, que la notion de bub énergétique serait devenue le leitmotiv de la politique énergétique turque. Issue d'une pensée dominante véhiculée par de nombreux 
rapports d'expertise ${ }^{1}$ et par des institutions financières internationales ${ }^{2}$, cette notion de bub énergétique appliquée au cas de la Turquie n'est que depuis très récemment l'objet d'une mise en débat dans les champs académiques turcs et étrangers ${ }^{3}$.

Aujourd'hui, nul ne peut contester le rôle géostratégique de la Turquie avec un territoire constituant une interface naturelle de transit entre les pays moyen-orientaux et caucasiens, détenteurs des plus grandes réserves mondiales de ressources fossiles, et le marché de consommation constitué par les pays européens. La diplomatie turque a su consolider et valoriser le rôle de corridor énergétique anatolien (flux pétroliers et gaziers), permettant à un pays en voie d'industrialisation et déjà majoritairement urbanisé - donc énergivore ${ }^{4}$ - de satisfaire sa demande intérieure tout en ayant la possibilité de diversifier ses sources d'approvisionnement. C'est dans cette perspective très géopolitique, autour de la «complexité des dépendances » entre différents types d'acteurs, que Pierre Chuvin, en 2008, se demandait si la Turquie allait devenir le futur hub énergétique de l'Europe ${ }^{5}$. Analyse que nous proposons d'actualiser dans une première partie à la lumière des nouvelles coopérations gazières avec l'Azerbaïdjan notamment, et au vu des tensions et conflits inhérents à la recomposition des intérêts en jeu dans une région marquée par de fortes instabilités politiques ces dernières années.

Toutefois, cet avantage géographique ne représente qu'une facette, certes indispensable, mais pas suffisante, pour prétendre définir la Turquie comme un hub. Derrière la polysémie du terme ${ }^{6}$, se cache égalementl'idée de plateforme infrastructurelle (stockage des ressources) et logistique (transbordement des ressources) permettant d'influencer les marchés énergétiques internationaux (emprise juridique sur la réexportation des ressources en transit sur le territoire) afin de disposer d'une capacité de régulation commerciale et financière de son marché intérieur (définition en temps réels des prix d'achat et de vente selon la variabilité de la demande). En ce sens et au delà des effets d'annonce, il y a consensus pour affirmer que l'on ne peut pas encore qualifier la Turquie de hub énergétique à l'échelle régionale et internationale ${ }^{7}$. La première raison invoquée concerne une dimension matérielle liée à l'insuffisance des capacités et de l'efficacité des infrastructures énergétiques du pays (mauvaise qualité des transmissions et de la distribution des flux, faible capacité de stockage...). La seconde raison relève de choix politiques invoquant la libéralisation incomplète des marchés énergétiques. Ce processus pourtant entamé au début des années 2000, n’a pas eu, aux 
Au delà du « hub » géopolitique, la régulation contestée du secteur énergétique en Turquie

yeux d'acteurs privés nationaux et internationaux, les effets escomptés en termes de volumes d'investissements privés dans les infrastructures de production et d'affaiblissement des anciens monopoles publics. Pire, le gouvernement AKP pourtant chantre de la libéralisation économique et de la privatisation des actifs de production ${ }^{8}$ depuis son arrivée au pouvoir en 2002, entraverait volontairement la libreconcurrence et l'auto-régulation des marchés énergétiques au titre de sa politique sociale. Ce dernier point, cause de plusieurs contradictions en termes de conséquences sociales (tarification, universalisation), mais aussi en termes de capacité de production (risque de pénurie d'approvisionnement), nous permettra légitimement dans un second temps de proposer un autre regard sur le bub énergétique turc.

\section{Turquie et géopolitique de l'énergie : un état des lieux}

Carrefour naturel entre les pays européens et le bassin Caspien, l'Anatolie constitue également une interface Nord-Sud entre la mer Noire et la Méditerranée. C'est ainsi que la Turquie s'est imposée au fil des constructions d'oléoducs, comme un corridor "Est-Ouest» privilégié, et est devenue, au début des années 1990, une pièce centrale du complexe puzzle de la « guerre des pipelines » ${ }^{9}$. Le ministère turc des Affaires Étrangères ${ }^{10}$ estime désormais que la Turquie assure environ $4 \%$ du transit du pétrole mondial grâce à l'oléoduc BakouTbilissi-Ceyhan mis en service en 2006, (il a transporté en 2012 jusqu’à 700000 barils par jour de pétrole azerbaïdjanais, kazakhe et turkmène) et à celui de Kirkouk-Yumurtalik ayant acheminé quotidiennement en 2011 environ 450000 barils de pétrole irakien. A ce flux terrestre, il faut également ajouter les 3 millions de barils quotidiens de pétrole russe et kazakhe qui transitent à travers les deux détroits maritimes de la Turquie (Les Dardanelles et le Bosphore) depuis le terminal de Novorossisk sur la mer Noire. Ainsi, renforcé depuis les récents débuts de l'exportation du pétrole "fédéral » du Kurdistan irakien ${ }^{11}$ - processus qui reste cependant fragile car contesté par le gouvernement central irakien ${ }^{12}-$ le complexe pétrochimique de Ceyhan s'affirme de plus en plus comme le véritable bub pétrolier de la Turquie. 


\section{Le gaz naturel au service de la diplomatie turque}

Les enjeux géopolitiques vis-à-vis du gaz naturel en Turquie - la seconde partie le révèlera de manière plus concrète - diffèrent en substance de ceux du pétrole car ils touchent à des dimensions sociétales plus massives et tangibles (production d'électricité et ressources utilisées pour le chauffage) si l'on omet l'exception du carburant automobile. Cette question du gaz naturel étant donc politiquement et socialement plus sensible, la Turquie n'a pas souhaité rester géopolitiquement « passive » en demeurant un simple pays de transit. Elle entend désormais devenir un bub gazier national afin de sécuriser et diversifier ses approvisionnements. C'est ainsi que, renforcée par sa désignation de $4^{\mathrm{e}}$ artère énergétique par la Commission Européenne (projet «Southern Gas Corridor ») qui souhaite se protéger des éventuelles sanctions énergétiques de la Russie, la Turquie s'est muée en acteur géopolitique actif depuis le début des années 2000.

Le levier énergétique est en effet devenu un instrument d'influence au service des intérêts de la nouvelle politique étrangère du gouvernement AKP arrivé au pouvoir en 2002, toute dédiée au renforcement du leadership régional du pays via une stratégie de rayonnement politique, économique et culturel. Preuve de ce nouveau poids diplomatique de la Turquie, Ankara avait conditionné sa participation au projet Nabucco ${ }^{13}$ aux avancées relatives à l'adoption du chapitre énergétique dans le cadre du processus d'adhésion à l'Union Européenne. Ankara s'était alors même permis de refuser la participation de Gaz de France au sein du consortium industriel en raison du soutien du gouvernement français de l'époque à la cause arménienne d'une part, et de son opposition affichée à l'entrée de la Turquie dans l'Union Européenne d'autre part ${ }^{14}$.

\section{Le TANAP : coalition turco-azerbaidjanaise, l'Union Européenne et la Russie}

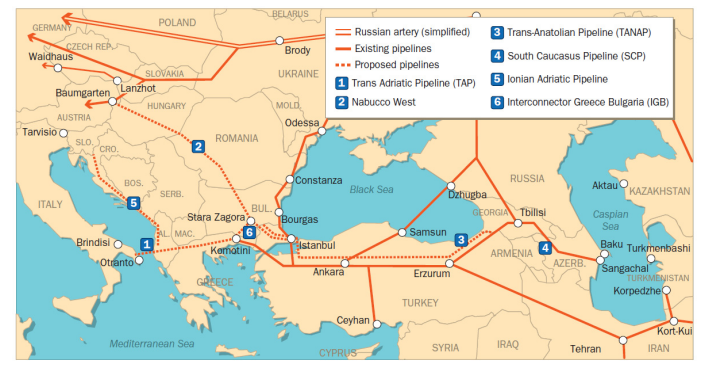

Source : Tagliapietra, S., (2014), 'Turkey as a Regional Natural Gas Hub: Myth or Reality? An Analysis of the Regional Gas Market Outlook, beyond the Mainstream Rhetoric', Nota di Lavoro 2.2014, Milan, Italy: Fondazione Eni Enrico Mattei, p.8 
Au delà du « hub » géopolitique, la régulation contestée du secteur énergétique en Turquie

Si l'adhésion institutionnelle de la Turquie à l'Union Européenne ne fait pas forcément l'unanimité, la concrétisation du projet TANAP a bénéficié de son soutien. Il est une preuve que le territoire turc est perçu comme une pièce stratégique de la sécurisation des flux énergétiques de l'Union Européenne. Il permet en effet de contourner géographiquement la dépendance au gaz russe. Le tracé du gazoduc trans-anatolien prévoit la traversée de 21 provinces turques, de la frontière géorgienne jusqu'en Bulgarie et en Grèce. Il deviendra ainsi la colonne vertébrale du corridor gazier sud-européen ${ }^{15}$, puisque les interconnexions seront ensuite possibles avec le TAP notamment, pour atteindre, via l'Italie, les marchés européens. Ce projet colossal, dont le coût est évalué à plus de 11 milliards de dollars, est porté et sera financé en majorité par la puissante compagnie nationale azérie, SOCAR $(70 \%)$ au côté de deux autres acteurs turcs, TPAO (20\%) et Botaş $(10 \%)$. Ce déséquilibre des responsabilités prises par les deux pays est surprenante quand on pense que le TANAP représente la pierre angulaire de la stratégie énergétique turque souhaitant devenir un bub gazier ${ }^{16}$. Un élément d'explication, jamais évoqué sérieusement au demeurant, devrait mettre en relation les orientations économiques du gouvernement turc au regard du montant des investissements directs de la SOCAR en Turquie dans des secteurs aussi variés que l'industrie pétro-chimique ou les médias ${ }^{17}$.

L'acheminement prévu de 31 milliards de $\mathrm{m}^{3}$ de gaz naturel prévus pour transiter via le TANAP en 2023 représente une menace potentielle sur l'influence régionale de la Russie. Toutefois, la Turquie reste un allié et demeure dépendante à court-terme de ses contrats d'approvisionnement via Gazprom mais aussi à long terme avec l'implication de l'entreprise Rosatom dans la construction de la $1^{\text {re }}$ centrale nucléaire turque. La Turquie coopère ainsi toujours avec la Russie pour la réalisation du projet South Stream - pourtant rival du projet TANAP - en l'autorisant à emprunter son espace économique exclusif en mer Noire.

Malgré la permanence de tensions diplomatiques, commerciales et militaires, toutes liées en partie à la pesanteur des différents héritages à l'origine de la formation des jeunes Etats-nations du Moyen-Orient et du Caucase, ce qui a pour conséquence de retarder l'achèvement des potentialités géo-énergétiques de la Turquie - citons le désaccord entre l'Azerbaïdjan et le Turkménistan autour Trans-capian Gas Pipeline prolongeant le TANAP jusqu'aux réserves turkmènes, les tumultes entre la Turquie, Chypre, Israël, la Palestine, le Liban autour de l'extraction 
des réserves off-shore du gaz Méditerranéen orientale ${ }^{18}$, ou encore la vulnérabilité accentuée des pipelines traversant la zone conflictuelle du Sud-Est turc depuis qu'ils servent de cibles à des actions de sabotages du PKK - la pression mondiale sur les hydrocarbures est telle que, si la Turquie réussit à maintenir la stabilité politique et économique dont elle jouit actuellement, ce pays devrait inévitablement renforcer son statut de territoire de transit, et de facto accroitre son poids diplomatique.

\section{Les défis de la nouvelle régulation du marché énergétique}

Pourtant, la Turquie, en tant que territoire d'intersection de ces flux énergétiques (le bub physique) ne perçoit pas uniquement l'enjeu énergétique sous l'angle du levier d'influence diplomatique (bub géopolitique). Cette proximité géographique des gisements d'hydrocarbures a été avant tout perçue depuis les années 1990, comme l'opportunité de soutenir une croissance économique, qui s'est nettement accélérée une décennie plus tard. Devenue une puissance émergente, de par son poids démographique et économique ${ }^{19}$, la Turquie est désormais en situation de forte dépendance envers ses voisins ${ }^{20}$. Les importations ${ }^{21}$ grevant de plus en plus la balance commerciale du pays, la stratégie énergétique a évolué et un nouveau type de régulation politique et économique a émergé au début des années 2000.

\section{La stratégie énergétique nationale: chimères politiques et risques de pénurie}

Réduire la dépendance énergétique est désormais l'un des objectifs prioritaire du ministère turc de l'Energie et des Ressources Naturelles. Pour cela, l'objectif fixé est de réduire la proportion de gaz naturel dans la production d'électricité de 45 à $30 \%$, compensée par un investissement massif dans les centrales hydro-électriques ${ }^{22}$ et les centrales thermiques alimentées en charbon ${ }^{23}$. Toutefois, sur la base d'études prospectives de consommation menées par Botaş en $2012^{24}$, cet objectif ne semble pas tenable. En effet, la demande va au minimum doubler d'ici 2030 passant de 45 à 81 milliards de $\mathrm{m}^{3}$. Citons deux facteurs contribuant à envisager une courbe de la demande toujours croissante malgré l'adoption de premières mesures d'efficacité énergétique. Tout d'abord, la demande 
Au delà du « hub » géopolitique, la régulation contestée du secteur énergétique en Turquie

d'électricité devrait continuer d'augmenter de 6 à 7\% chaque année jusqu'en $2023^{25}$. D'un autre côté, à l'échelle des plus grandes villes turques déjà connectées au réseau de gaz naturel, la politique de transformation urbaine initiée par le gouvernement turc permet l'accès au chauffage au gaz naturel à une frange de plus en plus importante de la population ${ }^{26}$.

Face à cette demande croissante, les pénuries d'approvisionnement et des coupures d'électricité que l'on croyait pourtant révolues en Turquie, menacent à nouveau le pays tout entier, tout en étant d'ores et déjà expérimentées épisodiquement et sporadiquement par une partie des citadins en période hivernale et estivale ${ }^{27}$. Le risque potentiel d'une crise énergétique généralisée à court et moyen terme est étroitement connecté à la temporalité géopolitique et aux statuts des contrats d'approvisionnements liant la Turquie à ses fournisseurs. L'importation, via le TANAP, des réserves azerbaijanaises du Shah Deniz II n'étant pas prévue avant 2018, la probabilité d'un premier sérieux déséquilibre entre demande et production dès 2015-2016 est élevée. Des raisons macro-structurelles contingentes au secteur énergétique turc accentuent ce risque. En effet, les capacités de stockage et le nombre de stations de compression de gaz naturel liquéfié sont largement insuffisants en Turquie, ce qui a par exemple contraint Botaş en 2008, 2009 et 2010 à ne pouvoir importer l'intégralité des volumes prévus par les contrats ${ }^{28}$. Pourtant, la nature des contrats Take-or-Pay ${ }^{29}$ oblige la Turquie à payer une quantité fixe de gaz qu'elle n'est pas en capacité d'importer et de consommer. À l'horizon 2015, aucun contrat n'est pour l'instant prévu pour accompagner la progression de la demande. Seule la Russie est en mesure de fournir des volumes plus conséquents mais la Turquie se montre réticente à acheter davantage aux prix en vigueur (environ $430 \$ / 1000 \mathrm{~m}^{3}$ ). En conséquence, à moyen et long terme, la Turquie souhaite alléger sa dépendance russe en important le gaz nord-irakien et azerbaïdjanais via le TANAP, dont les prix sont plus attractifs (environ $350 \$ / 1000 \mathrm{~m}^{3}$ ). Une seconde période de forte instabilité géopolitique après 2021-2022 est également à prévoir lorsque les contrats avec la Russie et l'Iran en vigueur aujourd'hui arriveront à terme.

\section{Libéralisme et interventionnisme : les effets rétroactifs d'une régulation énergétique contestée}

2001 fut une année charnière pour le secteur énergétique turc avec l'adoption de deux lois amorçant un processus de libéralisation des marchés de l'électricité et du gaz naturel ${ }^{30}$. La libéralisation de ces 
secteurs avait pour objectif de créer un marché compétitif, transparent et stable afin qu'une concurrence privée s'instaure pour satisfaire les besoins énergétiques du pays. La désintégration des différentes composantes du marché du gaz naturel, et la volonté de transférer le risque financier sur des opérateurs privés, ayant désormais légalement la possibilité d'intégrer les différents segments du marché (importation, transmission, commerce, production, distribution) devaient, entre autres, permettre la réduction drastique du poids des anciens monopoles étatiques, coupables désignés de la mauvaise gestion du secteur (gestion financière peu transparente, faible capacité de modernisation et d'universalisation des infrastructures, problème non résolu des taux de perte élevés liés aux captages illégaux).

Grâce au recul offert par plus de dix années écoulées, certains chercheurs ont mis en perspective les origines et les conséquences d'un processus tumultueux, non-linéaire et toujours inachevé ${ }^{31}$. L'une des principales conclusions de ces auteurs, est de montrer que loin de perdre la main sur un secteur politiquement stratégique, le gouvernement $\mathrm{AKP}$ continue de manière évidente de le réguler ${ }^{32}$. Cette situation est inévitablement source de conflits avec diverses institutions nationales et internationales partisanes du libre-échange.

Le premier point d'achoppement concerne le maintien d'une situation monopolistique de fait, de la part de Botaş, dans le secteur de la transmission et du commerce de gaz naturel ${ }^{33}$. Malgré l'objectif national officiel de réduire la part de marché de cette institution de $80 \%$ à $20 \%$, le ministère de l'Energie a paradoxalement soutenu et conforté le rôle de Botaş en la désignant agent principal d'un contrat d'approvisionnement de gaz naturel signé avec l'Egypte en 2008 et partenaire du TANAP.

Ce paradoxe fait pourtant sens si l'on conçoit Botaş comme la pierre angulaire permettant d'influencer à la fois les tarifs résidentiels du gaz naturel mais aussi ceux de l'électricité (celle-ci étant produite à presque $40 \%$ à partir de centrales thermiques privées). Un système de péréquation tarifaire ou, en d'autres termes, un système de subventionnement croisé - le secteur industriel subventionnant le résidentiel pour le gaz naturel résidentiel, et les provinces occidentales aisées subventionnant les taux de pertes supérieurs à $50 \%$ de certaines provinces de l'est du pays - a jusqu'à récemment permis d'atténuer, au profit des usagers résidentiels, les effets d'une augmentation des frais d'importation et des prix des matières premières, qu'un marché totalement dérégulé aurait reporté mécaniquement sur les factures ${ }^{34}$. Dans cette optique, 
Au delà du « hub » géopolitique, la régulation contestée du secteur énergétique en Turquie

la variable énergétique se trouve être une clé de compréhension de la longévité politique et du succès populaire de l'AKP, puisqu'elle participe pleinement de sa politique sociale redistributive et populiste ${ }^{35}$.

Pourtant, les premiers signes d'un retournement rétroactif d'une régulation énergétique ambivalente qui encourage la privatisation ${ }^{36}$ plutôt que la compétition ${ }^{37}$ et qui reste opportuniste car subordonnée à la temporalité des échéances politiques ${ }^{38}$, se font jour et seraient potentiellement dommageables en termes électoraux pour le gouvernement actuel. Les pressions internes et externes des élites économiques libérales ${ }^{39}$ ont conduit le gouvernement turc à renoncer - temporairement? - depuis début 2013 à cet interventionnisme. Une tarification d'électricité différenciée par régions, reportant directement sur l'usager les pertes financières en proportion du taux de captage illégal de chaque province, a été mise en place. Ceci devait in fine inciter les consommateurs à légaliser leur situation. Cette décision s'est traduit par une détérioration de la situation pour les sociétés privées de distribution actives dans les provinces du sud-est de la Turquie, se trouvant en grande difficulté de recouvrement suite à l'augmentation des impayés ${ }^{40}$. Conséquence directe ou contingente, ces compagnies sont à l'origine cet été de plusieurs coupures de courant, qui ont déclenché plusieurs protestations publiques ${ }^{41}$. La réponse politique qui s'en est suivie a été l'occasion de stigmatiser des fraudeurs de façon virulente : les arguments de «l'identité kurde » ont ainsi été superposés à une culture de l'illégalité instrumentalisée par le PKK. Ces comportements «déviants» sont qui plus est accusés de faire peser un fardeau financier supplémentaire sur les factures des consommateurs de l'ouest du pays ${ }^{42}$. Par ailleurs, l'hypothèse d'une généralisation des contestations sociales est forte, si l'on envisage les graves conséquences socio-économiques d'une dérégulation totale des prix de l'électricité et du gaz naturel sur une frange importante de la population et notamment celle en situation de pauvreté ${ }^{43}$.

En conclusion, la pertinence du concept de bub énergétique n'a de sens que si les dimensions physiques et géopolitiques qu'il recoupe sont analysées à l'aune des transformations internes du marché énergétique turc, en termes non seulement d'efficacité et de fiabilité d'approvisionnement, mais aussi en évaluant finement les effets socioéconomiques d'une libéralisation de la tarification sur les usagers vivant majoritairement en milieu urbain. Cette mise en perspective a pour conséquence de de nuancer la lénifiante prophétie de l'avènement proche du bub énergétique turc. 
Arik, Elvan. "Chronique d'une transition énergétique en tension: l'universalisation inachevée du réseau de gaz naturel à Istanbul ». Flux $n^{\circ}$ 93-94, $n^{\circ} 3$ (11 mars 2014) : 56-69.

Atiyas, I., et M. Dutz. " Competition and regulatory reform in the Turkish electricity industry ». In Prepared for presentation at the Conference on $\mathrm{EU}$ Accession, Turkey, Vol. 10, 2003.

Atiyas, Izak, Tamer Cetin, et Gurcan Gulen. Reforming Turkish Energy Markets: Political Economy, Regulation and Competition in the Search for Energy Policy. Springer Science \& Business Media, 2012.

Bagdadioglu, Necmiddin, Alparslan Basaran, Sibel Kalaycioglu, et Abuzer Pinar. Integrating Poverty in Utilities Governance. Ankara: UNDP Turkey CMEE Hacettepe Univeristy, 2009. http://www.tr.undp.org/content/dam/turkey/ docs/povreddoc/Integrating_Poverty_in_Utilities_Governance_ES.pdf.

Bagdadioglu, Necmiddin, Alparslan Basaran, et Catherine Waddams Price. Potential Impact of Electricity Reforms on Turkish Households. University of East Anglia CCP Working paper 07-8: ESRC Center for Competition Policy and Norwich Business School, 2007. http://papers.ssrn.com/abstract=993402. Bazin, Marcel, et Stéphane de Tapia. La Turquie: Géographie d'une puissance émergente. Armand Colin, 2012.

Bilgin, Mert. " Turkey's energy strategy: What difference does it make to become an energy transit corrdior, hub or center? ». UNISCI Discussion Papers, n 23 (2010): 113-28.

Bugra, Ayse, et Çaglar Keyder. New poverty and the changing welfare regime of Turkey. Ankara: UNDP, 2003. http://www.undp.org/content/dam/turkey/ docs/povreddoc/UNDP-TR-new_poverty.pdf.

Çetin, Tamer, et Feridun Yilmaz. "Transition to the Regulatory State in Turkey: Lessons from Energy ». Journal of Economic Issues 44, $n^{\circ} 2$ (1 juin 2010): 393-402. doi:10.2753/JEI0021-3624440211.

Cetin, T., et F. Oguz. " The reform in the Turkish natural gas market: A critical evaluation ». Energy policy 35, $\mathrm{n}^{\circ} 7$ (2007): 3856-67.

Chuvin, Pierre. "La Turquie : futur Hub énergétique de l'europe ?». Tiers Monde 194, $\mathrm{n}^{\circ} 2$ (2008): 359. doi:10.3917/rtm.194.0359.

Darbouche, Hakim, Bassam Fattouh, et Laura El-Katiri. « East Mediterranean Gas - what kind of a game-changer? ». Oxford Institute for Energy Studies, 2012. http://www.oxfordenergy.org/2012/12/east-mediterranean-gaswhat-kind-of-a-game-changer/.

Erdogdu, Erkan. "A review of Turkish natural gas distribution market ». Renewable and Sustainable Energy Reviews 14, n 2 (février 2010): 806-13. doi:10.1016/j.rser.2009.10.023.

Lussac, Samuel. "L'impossibilité du hub énergétique. La Turquie face aux limites de sa politique gazière ». EurOrient, L'Harmattan, nº 35-36 (2011). 0 uz, Fuat, K. Ali Akkemik, et Koray Göksal. « Can law impose competition? A critical discussion and evidence from the Turkish electricity generation market ». Renewable and Sustainable Energy Reviews 30 (février 2014): 381-87. doi:10.1016/j.rser.2013.10.024.

Pérouse, Jean-François. "La gouvernance énergétique dans les villes turques : un état des lieux ». Flux n 93-94, n 3 (11 mars 2014) : 70-76. Rzayeva, Gülmira. « Natural Gas in the Turkish Domestic Energy Market Policies and Challenges ». Oxford Institute for Energy Studies, 2014. http:// www.oxfordenergy.org/2014/02/natural-gas-in-the-turkish-domesticenergy-market-policies-and-challenges/.

Simonet, Loïc. "Les pipelines internationaux, vecteurs de prospérité, de puissance et de rivalités. Oléoducs et gazoducs dans la géopolitique et les 
Au delà du « hub » géopolitique, la régulation contestée du secteur énergétique en Turquie

relations internationales ». Revue internationale et stratégique $65, \mathrm{n}^{\circ} 1$ (1 mars 2007): 51-64. doi:10.3917/ris.065.0051.

Winrow, Gareth. « The Southern Gas Corridor and Turkey's Role as an Energy Transit State and Energy Hub ». Insight Turkey 15, n0 1 (2013). http://file. insightturkey.com/Files/Pdf/insight-turkey-vol_15_no_1_2013_winrow. pdf.

Notes

* Elvan Arik, doctorant en Urbanisme et Aménagement au sein du Labex IMU, programme de recherche POUDEV (Politiques et Usages De l'Energie en Ville). Chercheur associé au sein de l'Observatoire Urbain d'Istanbul (IFEA).

Elshan Mustafayev, doctorant en sciences politiques à l'IEP de Lyon. Affilié au GREMMO (Groupe de recherches et d'études sur la Méditerranée et le MoyenOrient) et à l'Antenne de Bakou de l'IFEA.

Ce travail a été réalisé grâce au soutien financier du LABEX IMU (ANR10-LABX-0088) de l'Université de Lyon, dans le cadre du programme " Investissements d'Avenir » (ANR-11-IDEX-0007) géré par l'Agence Nationale de la Recherche (ANR)

1. Voir par exemple le récent rapport commandité par The 0xford Institute for Energy Studies intitulé "Natural gas in the Turkish domestic energy market - Policies and challenges » et dont l'auteure, Gulmira Rzayeva, d'origine azerbaidjanaise, travaille au sein de nombreux think tank et centres de recherche internationaux comme le " Centre de recherche stratégique » rattaché au gouvernement azerbaidjanais (SAM) ou l'institut Hazard, financé par Socar, la compagnie pétrolière de l'Azerbaïdjan.

2. "Turkey to become global energy hub: World Bank director », http:// www.hurriyetdailynews.com/turkey-to-become-global-energy-hub-worldbank-director.aspx?pageID $=238 \& n I D=65605 \&$ NewsCatID $=348$, consulté le 12 août 2014

3. Mert Bilgin, «Turkey's energy strategy: What difference does it make to become an energy transit corrdior, hub or center? ", UNISCI Discussion Papers, n 23 (2010): 113-28; Samuel Lussac, « L'impossibilité du hub énergétique. La Turquie face aux limites de sa politique gazière », EurOrient, L'Harmattan, nº 35-36 (2011).

4. Depuis 1990, la consommation d'électricité a augmenté de $7 \%$ par an en moyenne jusqu'en 2010 Izak Atiyas, Tamer Cetin, et Gurcan Gulen, Reforming Turkish Energy Markets: Political Economy, Regulation and Competition in the Search for Energy Policy (Springer Science \& Business Media, 2012). et la consommation de gaz naturel de $15 \%$ par an pour atteindre 44,5 milliards de $\mathrm{m}^{3}$ Erkan Erdogdu, "A review of Turkish natural gas distribution market », Renewable and Sustainable Energy Reviews 14, n 2 (février 2010): 806-13, doi:10.1016/j.rser.2009.10.023.. Des prévisions à l'horizon 2030 tablent sur une consommation de gaz naturel comprise en 70 et 80 milliards de $\mathrm{m}^{3}$ Gülmira Rzayeva, «Natural Gas in the Turkish Domestic Energy Market Policies and Challenges », Oxford Institute for Energy Studies, 2014, http:// www.oxfordenergy.org/2014/02/natural-gas-in-the-turkish-domesticenergy-market-policies-and-challenges/..

5. Pierre Chuvin, « La Turquie : futur Hub énergétique de l'europe ? », Tiers Monde 194, n0 2 (2008): 359, doi:10.3917/rtm.194.0359. 
6. Les termes turcs de Koridor (corridor), Köprü (pont) qui apparaissent comme le $6^{\text {ème }}$ objectif de la version turque du Plan Stratégique 20102014 du Ministère des ressources naturelles et de l'énergie, sont traduits par hub dans la version anglaise illustrant ainsi l'inexistence d'une traduction littérale de ce terme dans la langue turque. On retrouve également le terme de terminal (terminal) énergétique utilisé pour désigner la Turquie mais qui dans les faits caractérise plus concrètement le complexe industrialoportuaire de Ceyhan, débouché méditerranéen du pétrole caucasien et dans une moindre mesure le port de Samsun sur la Mer Noire. Pour un meilleur aperçu des enjeux sous-jacents à la définition du terme de hub appliquée à la Turquie, se reporter à Lussac, (2011). " L'impossibilité du hub énergétique. La Turquie face aux limites de sa politique gazière ». EurOrient, (35-36)

7. Atiyas, Cetin, et Gulen, Reforming Turkish Energy Markets; Rzayeva, «Natural Gas in the Turkish Domestic Energy Market - Policies and Challenges ».

8. Jean-François Pérouse, "La gouvernance énergétique dans les villes turques: un état des lieux », Flux $n^{\circ} 93-94, n^{\circ} 3$ (11 mars 2014): 70-76.

9. Loïc Simonet, "Les pipelines internationaux, vecteurs de prospérité, de puissance et de rivalités. Oléoducs et gazoducs dans la géopolitique et les relations internationales », Revue internationale et stratégique $65, \mathrm{n}^{\circ} 1$ (1 mars 2007): 51-64, doi:10.3917/ris.065.0051.

10. http://www.mfa.gov.tr/turkiye_nin-enerji-stratejisi.tr.mfa

11. «Iraqi Kurdish oil arrives in Croatian port: Report », Hurriyet Daily News, 17/08/14, http://www.hurriyetdailynews.com/iraqi-kurdish-oil-arrives-incroatian-port-report.aspx?pageID $=238 \&$ nID $=70534 \&$ NewsCatID $=348$

12. Contestées par le gouvernement central de Bagdad, les modalités d'exportation du pétrole Nord-Irakien est centre des débats au moment de l'écriture de ces lignes : http://www.genelenergy.com/operations/ kurdistan-region-of-iraq.aspx

13. Le projet Nabucco - dont le tracé pressenti permettait d'extraire le gaz azéri jusqu'en Autriche et également soutenu par l'Union Européenne n'est désormais plus d'actualité ayant été supplanté par le choix du TANAP et le TAP.

14. Le changement de présidence française semble cependant avoir adouci les positions respectives, ce qui s'est notamment traduit par l'entrée d'Areva dans le consortium industriel de construction de la centrale nucléaire de Sinop en partenariat avec Mitsubishi.

15. Rzayeva, « Natural Gas in the Turkish Domestic Energy Market - Policies and Challenges »; Gareth Winrow, «The Southern Gas Corridor and Turkey's Role as an Energy Transit State and Energy Hub », Insight Turkey 15, $n^{\circ} 1$ (2013), http://file.insightturkey.com/Files/Pdf/insight-turkey-vol_15_no_1_ 2013_winrow.pdf.

16. Rzayeva, « Natural Gas in the Turkish Domestic Energy Market - Policies and Challenges ».

17. Voir Mustafayev E., "La Socar et les investissements directs de l'Azerbaïdjan en Turquie » posté le 23/11/2012 sur http://dipnot. hypotheses.org/27

18. Hakim Darbouche, Bassam Fattouh, et Laura El-Katiri, « East Mediterranean Gas - what kind of a game-changer? ", Oxford Institute for Energy Studies, 2012, http://www.oxfordenergy.org/2012/12/eastmediterranean-gas-what-kind-of-a-game-changer/.

19. Marcel Bazin et Stéphane de Tapia, La Turquie: Géographie d'une 
Au delà du « hub » géopolitique, la régulation contestée du secteur énergétique en Turquie

puissance émergente (Armand Colin, 2012).

20. Sur environ $90 \%$ d'énergies fossiles consommées par le pays, plus de $70 \%$ proviennent de l'extérieur. Environ $60 \%$ du gaz naturel consommé par la Turquie provient de la Russie et $20 \%$ de l'Iran.

21. Pour un ordre d'idée, en 2012, année plutôt stable économiquement, les dépenses énergétiques atteignaient 60 billions de dollars

22. Voir Arik. $E$, « Cartographie de l'installation des centrales hydroélectriques en Turquie », posté le 14/10/2013 sur http://dipnot.hypotheses.org/344

23. Voir Arik. E, «Tour d'horizon de l'actualité énergétique de la Turquie 2012-2013 », posté le 07/04/2013 sur http://dipnot.hypotheses.org/229

24. Rzayeva, « Natural Gas in the Turkish Domestic Energy Market - Policies and Challenges $»$.

25. D'ici là, les 3 centrales nucléaires (celle d'Akkuyu, de Sinop et la $3^{\mathrm{e}}$ centrale qui devrait être située sur les rives de la mer Noire à proximité de la frontière bulgare), censées assurer à moyen terme $10 \%$ de la production d'électricité nationale, ne seront pas encore en service

26. Des taux de croissance annuels de l'ordre de 12 à $13 \%$ sont en effet prévus par BOTAS jusqu'en 2030 (8-9 Bm3 aujourd'hui contre 22,7 Md3 en 2030)

27. De nombreuses coupures d'électricité liées à un défaut d'approvisionnement des centrales thermiques à gaz ont plongé des quartiers de plusieurs villes dans la pénombre et dans le froid et provoqué le mécontentement des habitants comme par exemple dans le quartier d'Esenyurt à Istanbul : http://www.todayszaman.com/news-333988central-istanbul-blackout-lingers-as-residents-grow-angry.html

28. Rzayeva, « Natural Gas in the Turkish Domestic Energy Market - Policies and Challenges ».

29. «Clause d'un contrat de fourniture de gaz ou d'électricité par laquelle le vendeur garantit la mise à disposition du gaz auprès de l'acheteur, qui garantit en contrepartie le paiement d'une quantité minimale d'énergie, qu'il en prenne livraison ou non » Source : http://www.cre.fr/glossaire/ clause-de-take-or-pay

30. I. Atiyas et M. Dutz, « Competition and regulatory reform in the Turkish electricity industry », in Prepared for presentation at the Conference on EU Accession, Turkey, vol. 10, 2003; T. Cetin et F. Oguz, " The reform in the Turkish natural gas market: A critical evaluation », Energy policy $35, \mathrm{n}^{0} 7$ (2007): 3856-67.

31. Atiyas, Cetin, et Gulen, Reforming Turkish Energy Markets; Tamer Çetin et Feridun Yilmaz, "Transition to the Regulatory State in Turkey: Lessons from Energy », Journal of Economic Issues 44, n⿳0 2 (1 juin 2010): 393-402, doi:10.2753/JEI0021-3624440211.

32. Atiyas, Cetin, et Gulen, Reforming Turkish Energy Markets; Fuat Oguz, K. Ali Akkemik, et Koray Göksal, «Can law impose competition? A critical discussion and evidence from the Turkish electricity generation market », Renewable and Sustainable Energy Reviews 30 (février 2014): 381-87, doi:10.1016/j.rser.2013.10.024.

33. Actuellement, seules 7 sociétés privées ont reçu l'autorisation d'importer directement du gaz russe en petite quantité $\left(9,75 \mathrm{Bm}^{3}\right)$. Du fait de leurs tailles respectives relativement réduite et de leur inexpérience sur le marché, les négociations auprès de Gazprom, plaçaient mécaniquement le géant russe dans une position de force qui n'a pas été sans dommages collatéraux pour Botas. L'arrivée de nouveaux acteurs privés sur le marché redéfinit profondément la nature des intérêts et des rapports de force 
Rzayeva, "Natural Gas in the Turkish Domestic Energy Market - Policies and Challenges ».

34. Atiyas, Cetin, et Gulen, Reforming Turkish Energy Markets; Çetin et Yilmaz, "Transition to the Regulatory State in Turkey ».

35. Elvan Arik, "Chronique d'une transition énergétique en tension: l'universalisation inachevée du réseau de gaz naturel à Istanbul », Flux $\mathrm{n}^{\circ}$ 93-94, n 3 (11 mars 2014) : 56-69 ; Ayse Bugra et Çaglar Keyder, New poverty and the changing welfare regime of Turkey (Ankara: UNDP, 2003), http://www.undp.org/content/dam/turkey/docs/povreddoc/UNDP-TRnew_poverty.pdf.

36. Sönmez M., "Energy becomes new love of large Turkish corporations », Hurriyet Daily News, 17/08/13, http://www.hurriyetdailynews.com/energybecomes-new-love-of-large-turkish-corporations.aspx?pageID= 238\&nID $=52689 \&$ NewsCatID $=348$

37. Atiyas, Cetin, et Gulen, Reforming Turkish Energy Markets.

38. Oguz, Akkemik, et Göksal, "Can law impose competition? ».

39. Vagliasindi, Maria. 2013. Implementing Energy Subsidy Reforms: Evidence from Developing Countries. Washington, DC: World Bank. $\odot$ World Bank. https://openknowledge.worldbank.org/handle/10986/11965 License: CC BY 3.0 IG0.

40. Selon Taner Yildiz, le Ministre de l'énergie, seul $10 \%$ des abonnés du sud-est de la Turquie s'acquitteraient de leur facture, représentant une perte totale de 4,9 milliards de livres. Voir Altunsoy I., " Electricity distribution firms in danger of seizure », Today Zaman, 10/08/14, http:// www.todayszaman.com/business_electricity-distribution-firms-in-dangerof-seizure_355159.html

41. Il a été reporté une manifestation pacifique dans la province de Sanliurfa ( Government, Kurdish politicians in row over energy bills », Hurriyet Daily News, 08/07/14, http://www.hurriyetdailynews. com/government-kurdish-politicians-in-row-over-energy-bills. aspx? pageID=238\&nID $=68836 \&$ NewsCatID $=348$ ). Des heurts plus violents ont également eu lieu cet été dans la ville du même nom lorsqu'une centaine de manifestants regroupés devant un transformateur électrique du centre ville pour dénoncer l'intensification des coupures de courant ont été délogés par les forces de l'ordre local. Voir « Elektrik isyanı çatısmaya dönüstü: Polis havaya ates açtı », Radikal, 16/08/2014, http://www. radikal.com.tr/turkiye/elektrik_isyani_catismaya_donustu_polis_havaya_ ates_acti-1207236

42. Surcoût annuel estimé à $140 \mathrm{TL}$ par ménage, voir « Minister targets PKK over illegal electricity use », Today Zaman, 08/08/14, http://www. todayszaman.com/news-352421-minister-targets-pkk-over-illegalelectricity-use.html

43. Necmiddin Bagdadioglu et al., Integrating Poverty in Utilities Governance (Ankara: UNDP Turkey CMEE Hacettepe Univeristy, 2009), http://www. tr.undp.org/content/dam/turkey/docs/povreddoc/Integrating_Poverty_ in_Utilities_Governance_ES.pdf; Necmiddin Bagdadioglu, Alparslan Basaran, et Catherine Waddams Price, Potential Impact of Electricity Reforms on Turkish Households (University of East Anglia CCP Working paper 07-8: ESRC Center for Competition Policy and Norwich Business School, 2007), http://papers.ssrn.com/abstract=993402. 